\title{
Evaluation économique d'un service d'approvisionnement dans la zone du Lac rose (Dakar, Sénégal) : exemple du maraichage
}

\author{
Thierno Bachir Sy \\ Laboratoire de Biogéographie \\ Département de Géographie \\ Université Cheikh Anta DIOP - Dakar - Sénégal
}

Sara Danièle Dieng

Unité de recherche sur l'environnement et les écosystèmes

Institut des Sciences de l'Environnement

Université Cheikh Anta DIOP - Dakar - Sénégal

\section{Amadou Tandjigora}

Laboratoire de recherche économique et monétaire

Faculté des Sciences Economiques et de Gestion

Université Cheikh Anta DIOP - Dakar - Sénégal

\author{
Edmée Mbaye \\ Laboratoire de Biogéographie \\ Département de Géographie \\ Université Cheikh Anta DIOP - Dakar - Sénégal
}

\begin{abstract}
Résumé : Cet article contribue à une meilleure connaissance de l'importance socioéconomique du maraichage au Lac Rose dans la zone périurbaine de Dakar. La méthodologie mixte (quantitative et qualitative) de collecte de données a été adoptée à cet effet. Elle a permis d'effectuer une analyse descriptive de la situation socioéconomique des acteurs maraichers.

Les résultats révèlent une grande importance de cet écosystème lacustre parce que participant activement dans l'approvisionnement en produits maraichers pour la population locale et le marché urbain dakarois. En plus, le maraichage garantit des revenus substantiels aux exploitants qui s'y adonnent. Le revenu moyen par maraicher sur une récolte est de 893750 F FCA (1 375 Euro) tandis que le revenu plus élevé sur une récolte est 3000000 F CFA (4 615,3 Euro). Cet article montre un fort intérêt socioéconomique de l'écosystème du Lac Rose, ce qui doit amener à préserver cet espace naturel de l'urbanisation galopante en cours dans la zone.
\end{abstract}

Mots-clés : Services écosystémiques - Services d'approvisionnement - Lac rose - Maraichage Urbanisation - Niayes - Sénégal.

Digital Object Identifier (DOI): https://doi.org/10.52502/ijesm.v1i1.160 


\section{Introduction}

Les écosystèmes naturels fournissent des services vitaux aux humains. Ils sont à la base de toute possibilité de vie, de production (nourriture, boisson, énergie etc.) et de reproduction humaine aussi bien dans les milieux ruraux qu'urbains (Serpantié et al. 2012 ; Millenium Ecosystem Assesment-MEA, 2005). Ces services écosystémiques sont classifiés sous 4 types principaux (MEA, 2005) : les services d'approvisionnement, les services de régulation, les services support et les services culturels. Le fond mondial pour la nature (WWF) estimait en 2004 la valeur annuelle des zones humides dans le monde à 70 milliards de dollars US (Dujin et al., 2008).

Dans le même sillage, les Etats africains semblent conscients de l'importance de l'évaluation des services écosystémiques et commencent à l'intégrer dans leur politique environnementale et/ou de développement durable. A titre d'exemple, le Cameroun a validé en 2018 son rapport national d'évaluation des écosystèmes.

Au Sénégal, plusieurs contributions à l'évaluation des services fournis par les écosystèmes sont effectuées ces dernières années (Ngom et al. 2014, Diatta et al. 2016 ; Badiane et Mbaye, 2018). Ces études ont concerné tous les types d'espaces - ruraux, urbains, périurbains - et les composantes biotiques et abiotiques du capital naturel du pays.

Dans une étude sur la réserve de biosphère du Ferlo au Centre Nord du Sénégal, Ngom et al. (2014) identifient 6 services d'approvisionnement fournis aux populations locales qui sont entre autres : la nourriture, le fourrage, la pharmacopée, les bois d'énergie, de construction et d'artisanat. Toujours dans cette perspective de contribuer à la connaissance de la valeur des services écosystémiques offerts par la composante végétale, Dieng et al. (2016) ont réalisé une étude sur l'espèce Cordyla pinnata dans la forêt classée de Patako et ses périphéries au Centre Ouest du Sénégal). Les auteurs notent par exemple que le Cordyla pinnata fournit 20 services écosystémiques aux populations riveraines dont les principaux services d'approvisionnement identifiés sont : la nourriture, le bois d'énergie, le bois de service et les produits médicinaux.

Par ailleurs, s'interrogeant sur la situation des zones humides c'est-à-dire les opportunités ou menaces qui peuvent en découler - précisément pour la zone des Niayes de Dakar - Badiane et Mbaye (2018) ont révélé qu'un exploitant peut glaner un gain moyen annuel de 5000000 F CFA (7 634 Euros) au Technopôle.

En effet, les Niayes constituent un espace éco géographique particulier, marqué par une succession de lacs inter-dunaires (lac Tanna, lac rose, Mbeubeuss, Wouye, Thiourour, Warouwaye, etc.) le long de la grande côte sénégalaise (Sène et al. 2018). Dans ce lot, le lac Retba ou lac rose est d'une spécificité très remarquable. Il attire des milliers de visiteurs par an, de par sa coloration rose. Aussi, sa forte salinité 10 fois plus élevée que celle de l'eau de mer, permet depuis plusieurs décennies la production artisanale de sel. Il s'y ajoute, tout autour, l'existence de périmètres maraichers. Ainsi, l'importance de ses services culturels et d'approvisionnement garantit des revenus substantiels aux exploitants.

Néanmoins, des études (Sow, 2012 ; Diallo, 2010 ; Sène, 2009) ont fait état de mutations importantes dans les espaces périurbains de Dakar, notamment la zone du lac rose. Cette dernière, plébiscitée par les autorités publiques du Sénégal pour désengorger Dakar, doit abriter le second pôle urbain de la future agglomération de Diamniadio (Dakar et environ, Horizon 2035). Ainsi, la spéculation foncière y va bon train. Cette situation, combinée aux phénomènes des changements climatiques, doit nécessairement susciter des interrogations sur les enjeux et les potentialités des zones humides dans les milieux urbains. 
Le présent article vise à contribuer à une meilleure connaissance de la valeur économique des services écosystémiques fournis par le lac rose et sa périphérie, notamment les services d'approvisionnement. La première partie porte sur l'identification des acteurs bénéficiaires de la culture maraichère. Quant à la seconde, elle procède à une évaluation de la valeur économique de l'activité maraichère.

\section{Présentation de la zone d'étude}

La zone retenue pour cette étude est le périmètre maraicher du lac rose, situé dans le village de Bonabé. Ce choix est lié d'abord à son caractère écosystémique assez spécifique. Il appartient en effet, à la région éco géographique des Niayes. Le deuxième élément concerne le fait que ces dernières années, d'importantes mutations en lien avec l'urbanisation y sont notées. Ces mutations semblent impacter l'écosystème dans la prestation de services écosystémiques. Sur le plan géographique, la zone du lac rose se situe entre les coordonnées $14^{\circ} 50$ Nord et $17^{\circ} 15$ Ouest. (Figure 1).

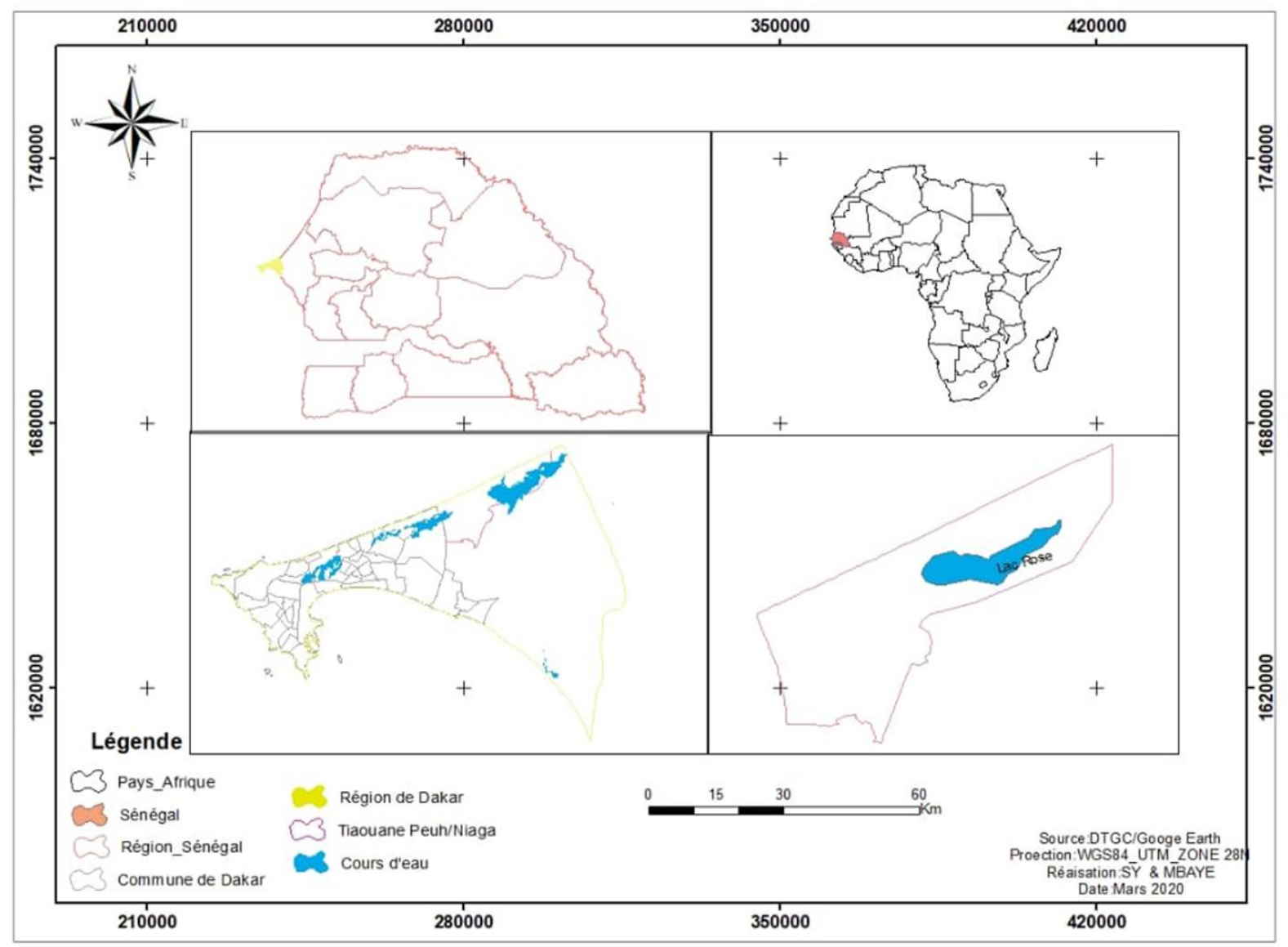

Figure 1 : Localisation de la zone d'étude

Au demeurant, la zone d'étude est soumise à un processus d'urbanisation accéléré qui se répercute sur le rétrécissement des zones de culture au profit des zones d'habitation (Figure 2). 

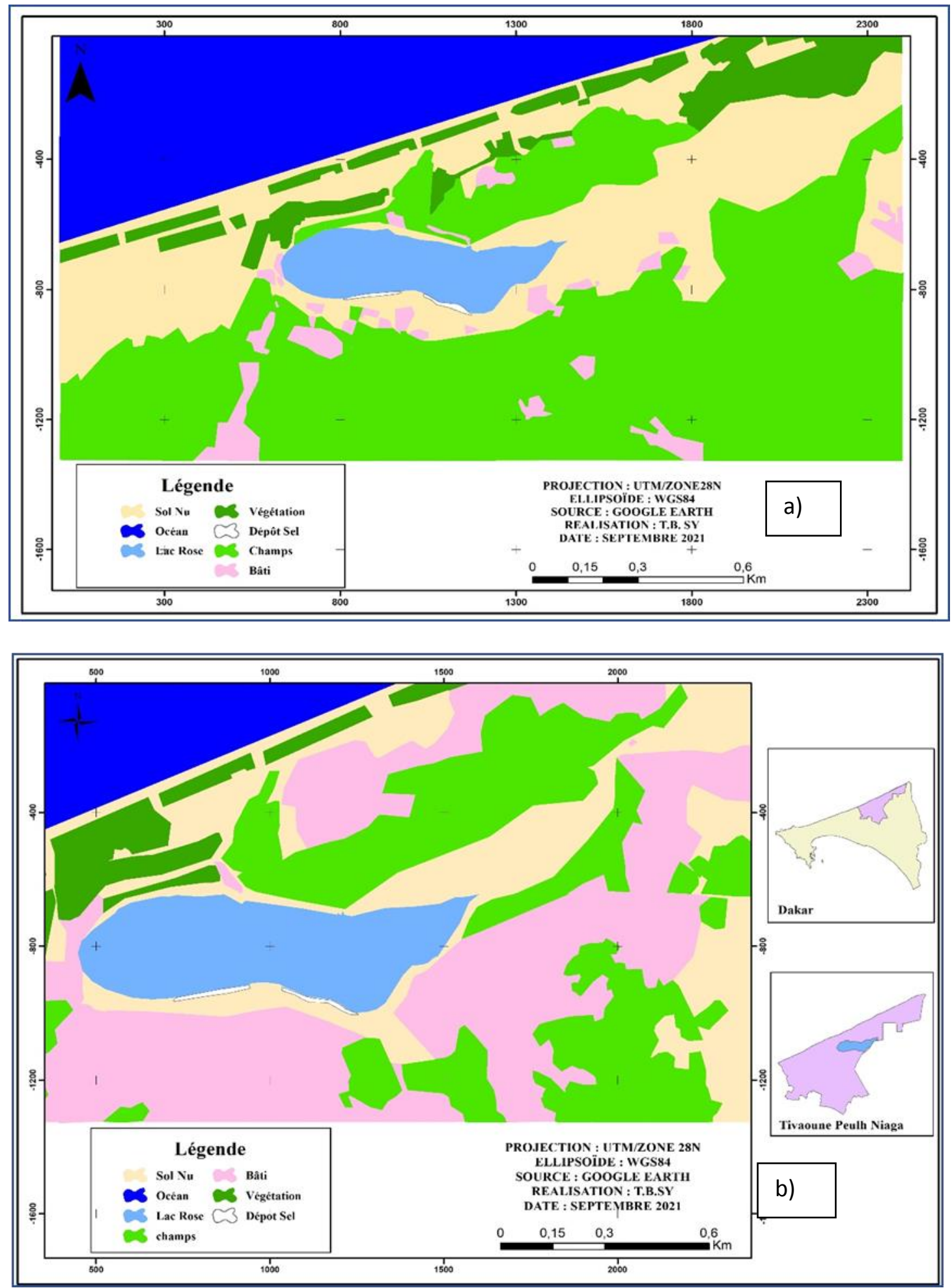

Figure 2 : modification de l'occupation du sol dans la zone du lac rose entre 2008 (a) et 2019 (b)

\section{Méthodologie}

- Echantillonnage et collecte de données

Une visite exploratoire préalablement effectuée a permis d'entrer en contact avec les acteurs maraichers et de peaufiner le questionnaire. Ce dernier a constitué le principal outil de collecte de données. Il a été administré de manière aléatoire à trente (30) exploitants du village de Bonabé. Chacun des exploitants gère à titre personnel un périmètre qui lui est propre. 
Le questionnaire a permis de recueillir des données qualitatives et quantitatives sur l'activité maraichère. Il avait trait à l'identification des acteurs, les spéculations, l'évaluation financière et le consentement à payer. Ce dernier nécessite la présentation d'un scénario pour recueillir des informations pertinentes par le biais des enquêtes. Il peut s'opérer par deux mécanismes (CGDD/SEEIDD, 2010) : soit le consentement à payer (c'est-à-dire la tendance d'un agent à débourser une somme d'argent pour continuer à profiter d'un service) soit la disposition à accepter (ce qui veut dire l'indemnisation ou le dédommagement que reçoit un acteur pour un service perdu). Dans le cas d'espèce nous avons choisi la première option, en proposant comme scénario une urbanisation galopante pouvant engendrer une cessation de la production maraichère dans la zone du lac rose. Ce qui interpelle les exploitants face à une telle situation de donner un montant qu'ils sont prêts à payer pour continuer à bénéficier de l'écosystème du lac pour la pratique de leurs activités.

En outre, des entrevues ont aussi été organisées avec certaines personnes ressources. L'objectif de ces dernières est de compléter d'une part les informations fournies par les données quantitatives et d'autre part de comprendre la dynamique agricole dans la zone.

\section{- Traitement des données}

Le dépouillement des questionnaires a été effectué sur Excel. Ce qui a permis de disposer de données pour le traitement statistique. Après correction des données saisies sur Excel, le logiciel $\mathrm{R}$ a été utilisé pour l'analyse statistique. Ce qui a permis d'avoir des variables multicritères. L'analyse statistique a concerné les citations exprimées par les informateurs, les revenus des exploitants et le consentement à payer.

\section{Résultats et discussion}

\subsection{Les acteurs du maraichage}

Le maraichage constitue une des principales activités socioéconomiques dans la zone du lac rose. Le maraichage y est assez dynamique, par ce qu'il peut durer toute l'année. En plus, il emploie un nombre d'individus non négligeable. Les exploitants maraichers sont surtout des jeunes pour la plupart âgés entre 17 et 35 ans. Cette tranche d'âge correspond à $60 \%$ des maraichers enquêtés dans la zone. En plus, la proportion des adultes dont la tranche d'âge est comprise entre 35 et 50 ans est de $30 \%$. Les jeunes et les adultes représentent ainsi $90 \%$ des maraichers. Cette situation s'explique par l'utilisation de moyens rudimentaires pour l'activité maraichère. Ce qui requiert une force physique pour optimiser les capacités de production.

D'autre part, ce secteur pourrait constituer un pourvoyeur d'emplois aux jeunes non seulement de Dakar, mais aussi pour les autres régions du pays et la sous-région (Sidy et Zelem, 2010). En outre, il n'exige pas un niveau élevé de qualification pour les chercheurs d'opportunités venus de l'intérieur du pays et des Etats voisins. A ce titre, parmi les informateurs, les exploitants maraichers originaires de la Guinée Conakry sont majoritaires à 53,3\% tandis que les sénégalais constituent $46 \%$.

Toutefois, la forte présence des maraichers issus de la sous-région amène à s'interroger sur le mode d'acquisition des périmètres de culture. En ce sens, 3 scénarii sont distingués dans l'acquisition des terres destinées à la culture maraichère. D'abord, il existe la location des champs moyennant une somme d'argent ou par le métayage. Ensuite, il y a le recrutement de d'ouvriers agricoles. Pour ce faire, le propriétaire foncier engage un exploitant maraicher et assure l'ensemble des investissements pour la culture de même que son alimentation jusqu'à la récolte des produits. Après la commercialisation, il récupère le montant des investissements réalisés. Le restant des recettes est partagé en deux parts égales entre le propriétaire et l'exploitant. Enfin, même si la loi nº64.46 de 1964 sur le domaine national du Sénégal stipule que la terre est une propriété de l'Etat qui l'attribue à qui voudrait la mettre en valeur, l'achat des parcelles culturales est le plus souvent effectué par des gens appartenant à la classe moyenne. 
Ces derniers décident généralement de construire des habitations ou simplement de laisser les terres en hibernation. Ce qui contribue à la rareté des espaces cultivables.

Sous un autre angle, Les hommes sont omniprésents dans ce secteur avec 97\% des exploitants. En plus du problème d'accessibilité aux terres cultivables, il s'y ajoute le caractère rudimentaire de l'activité qui est difficilement associable aux travaux ménagers dont les femmes sont responsables, dans pour la plupart des cas (Sène, 2009).

Pour ce qui est des structures d'appui, 2 organisations de producteurs maraichers intervenant dans la zone d'étude, ont été identifiées. Il s'agit de l'Association des Unions Maraichères des Niayes (AUMN) et de l'Association Nationale des Horticulteurs du Sénégal (ANDH). Celles-ci assistent les travailleurs dans l'organisation effective des activités de maraichage.

\subsection{L'organisation de l'exploitation maraichère}

Les populations de la zone du lac rose ont commencé à s'intéresser à la culture maraichère depuis la sécheresse des années 1970 qui a vu la production des céréales vivrières, fortement baisser. En outre, le maraichage est plus adapté aux besoins de subsistances des ménages parce que les légumes peuvent facilement être commercialisés mais aussi l'écosystème est adapté à cette culture qui est praticable pendant toute l'année.

L'estimation de la superficie emblavée par les 30 maraichers interrogés dans le cadre de la présente étude fait état de 23, 97 ha, soit en moyenne 0,8 ha par producteur. La taille du périmètre maraicher maximal est de 2,5 ha contre 0,05 ha pour la taille minimale. Cette situation dénote d'une forte présence des exploitations individuelles. En effet, selon Touré et Seck (2005), les exploitations individuelles sont souvent principalement caractérisées, dans la zone des Niayes, par l'étroitesse des périmètres (moins d'1ha).

Les principaux produits maraichers cultivés dans la zone sont le chou pommé (Brassica oleracea L.), le navet (Brassica napus L.), l'oignon (Allium cepa L.), la carotte (Daucus carota L.), le persil (Petroselinum crispum), le poivron (Capsicum annuum), la menthe (Mentha), le gombo (Abelmoschus esculentus) et la tomate (Solanum lycopersicum). Le navet est la principale spéculation dans la zone et totalise $32,5 \%$ des citations (voir Tableau 1).

Tableau 1: Produits maraichers en fonction de la fréquence des citations

\begin{tabular}{|l|l|l|}
\hline Produits maraichers & Nombre de citations & Fréquence des citations en \% \\
\hline CHOU POMME & 18 & 21,6 \\
\hline NAVET & 27 & 32,5 \\
\hline OIGNON & 5 & 6 \\
\hline CAROTTE & 10 & 12 \\
\hline PERSIL & 6 & 7,2 \\
\hline POIVRON & 9 & 11 \\
\hline GOMBO & 2 & 2,4 \\
\hline TOMATE & 2 & 2,4 \\
\hline MENTHE & 4 & 4,8 \\
\hline TOTAL & $\mathbf{8 3}$ & $\mathbf{9 9 , 9}$ \\
\hline
\end{tabular}

Source : Donnée de terrain Sy, Janvier 2019 
En effet, 5 récoltes sont réalisées en moyenne par exploitant sur une année. Néanmoins, il a été constaté la pratique de plusieurs cultures sur un même périmètre. Cette pratique se fait souvent de deux manières. La première consiste à maintenir une culture principale avec une durée plus longue et des cultures secondaires qui permettront d'engranger des revenus intermédiaires. C'est généralement le cas de la culture du chou pommé.

La seconde manière concerne la division du champ en deux parties et la rotation de deux cultures sur chacune des parcelles. Cette pratique est une alternative au manque de jachère que leur impose l'étroitesse des périmètres maraichers (Faye, 2010).

Par ailleurs, 3 principaux usages sont faits de la production de légumes dans la zone d'étude. C'est d'abord essentiellement la vente, ce qui permet aux exploitants d'avoir un revenu en fonction des récoltes. Ensuite, il s'agit de l'utilisation dans l'alimentation des ménages des exploitants et enfin les dons. Ces deux dernières formes d'utilisation font de la production maraichère une source d'approvisionnement vitale pour la nourriture notamment en légumes.

Le Marché Thiaroye dans la banlieue dakaroise, constitue le principal lieu d'écoulement (73,3\% des citations) des produits maraichers issus de la zone du lac rose. En effet, les exploitants maraichers font souvent appel à des intermédiaires (coxeurs ${ }^{1}$ ) qui les aident à écouler leurs produits. Après la vente des produits, ces intermédiaires sont rémunérés soit en fonction d'un montant fixé par les deux parties soit par le surplus obtenu sur le prix de vente du sac, qui est défini à l'avance. D'ailleurs, des revendeurs (généralement des femmes banabanas ${ }^{2}$ ) sillonnent les périmètres maraichers pour acheter des légumes, pour ensuite aller les écouler sur les marchés. Toutefois, cette pratique semble baisser $(36,6 \%$ des citations) du fait que les maraichers ont commencé à découvrir qu'acheminer les produits au marché est beaucoup plus rentable que de les écouler sur place.

\subsection{Evaluation économique du maraichage}

L'étude des revenus des exploitants maraichers est fortement soumise à la durée des cultures, au type de spéculation, à la période de l'année, à la réalité des marchés, ... Ainsi, le revenu des maraichers émane principalement de la commercialisation des produits après récolte. En moyenne, la vente des produits rapporte $893750 \mathrm{~F}$ FCA (1 375 Euro) par exploitant maraicher et par récolte. Le revenu minimal par récolte est $45000 \mathrm{~F}$ CFA (69,2 Euro) tandis que celui maximal pour une récolte, est à 3 000000 F CFA (4 615,3 Euro). Selon Diop et al. (2019), pour ce qui est de la zone humide du technopôle de Dakar, le revenu mensuel des maraichers est compris entre 120000 F CFA et 800000 F CFA. Ce revenu évalué mensuellement ne semble pas adapté au maraichage qui rémunère ses acteurs à travers la vente des récoltes. Toutefois, le revenu des maraichers peut être estimé annuellement. Badiane et Mbaye (2018), ont défini le revenu moyen annuel des maraichers des lacs Wouye et Mbeubeuss, respectivement à $2000000 \mathrm{~F} \mathrm{CFA}$ et $800000 \mathrm{~F} \mathrm{CFA}$.

Cette forte différence peut s'expliquer par les tailles inégales des surfaces emblavées mais aussi par le type de spéculation cultivée (fortement dépendant des moyens à mobiliser et de la durée du cycle de production). Sur ce dernier point, l'exploitant maraicher présentant le revenu minimal par exemple, ne présente pas d'importants investissements mis à part les coûts des semences et de l'engrais (5 $000 \mathrm{~F}$ CFA par récolte). C'est ce qui permet d'affirmer que la production maraichère et par de là, le revenu des exploitants est en fonction de la capacité d'investissement, en plus de la disponibilité des terres foncière adéquate.

Les principaux coûts liés à la production maraichère, au-delà des intrants agricoles, des semences et des engrais, sont la main d'œuvre, le bail foncier et le carburant (l'essence) utilisé pour les pompes à eau. L'importance des coûts liés à la main d'œuvre et à la location des terres $(69,8 \%$ des citations, les deux combinés) témoigne aussi de la forte présence des étrangers ou des personnes non-autochtones. Les

\footnotetext{
${ }^{1}$ Grossiste chargé de l'écoulement des produits dans les marchés

${ }^{2}$ Commerçants itinérants qui collectent les produits des zones de production aux zones de commercialisation
} 
dépenses moyennes sur une récolte sont de 234216 F CFA (360,3 Euro), avec un pic de 750000 F CFA (1 153,8 Euro).

Le bénéfice moyen tiré des exploitations maraichères s'élève à 659533 F CFA (1 014,7 Euro). Le plus faible bénéfice égale $40000 \mathrm{~F}$ CFA (61,5 Euro) tandis que le plus important est $2500000 \mathrm{~F}$ CFA (3 846 Euro).

La culture de l'oignon enregistre le plus important bénéfice par récolte (tableau 2) tandis que celle du persil est la moins rentable. Il faudrait intégrer à cet élément d'analyse la durée des cultures pour voir celle qui rapporte plus d'argent sur une base mensuelle.

Tableau 2 : Principale spéculation, revenus, dépenses et bénéfices des exploitants maraichers sur une récolte

\begin{tabular}{|c|c|c|c|c|}
\hline Acteurs & Principale spéculation & Revenus par récolte & Dépenses par récolte & Bénéfice \\
\hline 1 & Persil & 1600000 & 600000 & 1000000 \\
\hline 2 & Navet & 500000 & 50000 & 450000 \\
\hline 3 & Navet & 375000 & 45000 & 330000 \\
\hline 4 & Chou & 1400000 & 750000 & 650000 \\
\hline 5 & Persil & 200000 & 19000 & 181000 \\
\hline 6 & Navet & 1200000 & 302000 & 898000 \\
\hline 7 & Chou & 1200000 & 85000 & 1115000 \\
\hline 8 & Persil & 250000 & 71000 & 179000 \\
\hline 9 & Navet & 1680000 & 600000 & 1080000 \\
\hline 10 & Chou & 1600000 & 250000 & 1350000 \\
\hline 11 & Persil & 279000 & 170000 & 109000 \\
\hline 12 & Navet & 247000 & 150000 & 97000 \\
\hline 13 & Chou & 450000 & 264000 & 186000 \\
\hline 14 & Navet & 450000 & 75000 & 375000 \\
\hline 15 & Navet & 962500 & 450000 & 512500 \\
\hline 16 & Navet & 750000 & 200000 & 550000 \\
\hline 17 & Persil & 364000 & 70500 & 293500 \\
\hline 18 & Navet & 300000 & 30000 & 270000 \\
\hline 19 & Oignon & 3000000 & 500000 & 2500000 \\
\hline 20 & Navet & 1260000 & 200000 & 1060000 \\
\hline 21 & Oignon & 45000 & 5000 & 445000 \\
\hline 22 & Navet & 2000000 & 300000 & 1700000 \\
\hline 23 & Oignon & 2000000 & 100000 & 1900000 \\
\hline 24 & Chou & 400000 & 150000 & 250000 \\
\hline 25 & Chou & 350000 & 150000 & 200000 \\
\hline 26 & Navet & 450000 & 250000 & 200000 \\
\hline 27 & Navet & 300000 & 30000 & 270000 \\
\hline 28 & Navet & 500000 & 160000 & 340000 \\
\hline 29 & Navet & 1500000 & 500000 & 1000000 \\
\hline 30 & Navet & 1200000 & 500000 & 700000 \\
\hline \multicolumn{2}{|r|}{ Moyenne } & 893750 & 234217 & 659533 \\
\hline
\end{tabular}

Source : Donnée terrain Sy, Janvier 2019

Par ailleurs, le pourcentage des informateurs maraichers ayant donné une valeur pour le consentement à payer est de $26,7 \%$. Il est à préciser que les valeurs exprimées concernent une récolte pour leur permettre de continuer à cultiver leurs champs, indépendamment de la culture et de la taille du champ. Le consentement à payer moyen exprimé par les maraichers, par récolte, égale 15781 F CFA (24.3 Euro). 
Le consentement à payer le plus faible donné est 15000 F CFA (23 Euro) alors que le plus élevé est de 50000 F CFA (77 Euro).

\section{Conclusion et recommandations}

Cette étude a mis en exergue l'importance socio-économique du lac rose et sa périphérie, à travers l'activité du maraichage qui emploie un nombre important de jeunes. Celle-ci produit une importante quantité de légumes destinée principalement à approvisionner les marchés de Dakar. Ainsi, la commercialisation garantit aux exploitants maraichers une source de revenus non négligeable. En moyenne, ils en tirent un revenu par récolte de 893750 F FCA. De même, les produits maraichers récoltés entrent dans la consommation des ménages de la zone.

Malgré ce fort intérêt du site, l'écosystème du lac rose demeure fortement menacé par l'avancé du front urbain, réduisant drastiquement les espaces culturaux. En effet, le plan d'urbanisme Dakar et environs, horizon 2035, a retenu la zone du lac rose pour le pôle secondaire du projet urbain de Diamniadio, nouvel axe de l'agglomération dakaroise. Cet élan nouveau de l'urbanisation s'accompagne de la construction d'importantes infrastructures (Aéroport International Blaise Diagne, Autoroute à péage, Voie de Dégagement Nord, Train Express Régional) qui favorisa à son tour la ruée des populations vers cette zone dans l'acquisition de parcelles pour divers usages. Mais le plus important demeure la construction d'habitats. Cette montée de l'espace bâti se fait principalement au détriment des parcelles de cultures.

Enfin, il existe des constructions aux abords du lac rose, à environ moins de 10 mètres du plan d'eau. Même si la prise en compte des spécificités des Niayes en particulier et des écosystèmes naturels en général, est un objectif du plan directeur d'urbanisme (Dakar et environs, horizon 2035), une urbanisation non contrôlée dans la pratique serait calamiteuse pour la zone. Ainsi, cette situation, combinée aux effets néfastes des changements climatiques, accélérerait le tarissement de cette lagune sur-salée et entrainerait des inondations comme celles connues récemment dans plusieurs zones de la banlieue de Dakar.

\section{BIBLIOGRAPHIE}

[1] S. D. Badiane et E. Mbaye. Zones humides urbaines à double visage à Dakar : opportunité ou menace ? Revue des Sciences Eaux \& Territoires - article hors-série $n^{\circ}$ 51. 2018.

[2] CGDD/SEEIDD. Donner une valeur à l'environnement : la monétarisation, un exercice délicat mais nécessaire. La Revue du Service de l'économie, de l'évaluation et de l'intégration du développement durable. 88 pages. www.developpement-durable.gouv.fr

[3] S. Diallo. La périurbanisation dakaroise : analyse d'impact dans la zone rurale de Sangalkam. Mémoire de Master, Géographie, FLSH, UCAD, Dakar, 85 pages. 2010

[4] A. A. Diatta, N. Ndour, A. Manga, B. Sambou , C. S. Faye, L. Diatta, A. Goudiaby, C. Mbow, S. D. Dieng. Services écosystémiques du parc agroforestier à Cordyla pinnata (Lepr. ex A.Rich.) Milne-Redh dans le Sud du Bassin Arachidier (Sénégal). International Journal of Biological and Chemical Sciences, vol.10, nº6, p. 2511-2525. (2016).

[5] S. D. Dieng, M. Diop, A. Goudiaby, F. N. Diop, L. C. Faye, I. Guiro, S. S Ambou, A. Lykke, B. Sambou. Caractérisation des services écosystémiques fournis par Cordyla pinnata dans la périphérie de la Forêt classée de Patako au Sénégal », VertigO - la revue électronique en sciences de l'environnement, Vol.16, n². (2016)

[6] Millennium Ecosystem Assessment-MEA. "Ecosystems and human well-being." Synthesis. 36 p. (2005).

[7] D. Ngom, M. C. Mohamed, O. Sarr, A. Bakhoum et E. A. Léonard. Perceptions communautaires sur les services écosystémiques d'approvisionnement fournis par le peuplement ligneux de la Réserve de Biosphère du Ferlo (Sénégal). VertigO - la revue électronique en sciences de l'environnement [En ligne], Volume 14 (2). (2014)

[8] L. Sène. Approche d'une géographie par le genre : la division du travail au lac Rose et au centre de pêche de Kayar (Sénégal). Mémoire de maitrise de Géographie, UCAD, Dakar. (2009).

[9] A. Sène, M. A. Sarr, A. Kane, et M. Diallo. L'assèchement des lacs littoraux de la grande côte du Sénégal : Mythe ou réalité ? Cas des lacs Thiourour, Warouwaye et Wouye de la banlieue de Dakar. J. Anim. Plant Sci, 35, 56235638. (2018). 
[10] G. Serpantié, P. Méral et C. Bidaud. Des bienfaits de la nature aux services écosystémiques : Éléments pour l'histoire et l'interprétation d'une idée écologique. VertigO : la revue électronique en sciences de l'environnement, 12(3). (2012).

[11] P. Sow. Uncertainties and conflicting environmental adaptation strategies in the Region of the Pink Lake, Senegal. ZEF Working Paper 101. Bonn. Germany. (2012).

[12] M. C. Zelem et T. Sidy. La valorisation environnementale et économique de " déchets" organiques dans le maraîchage périurbain à Dakar au Sénégal : acteurs, opportunités, contraintes et risques. FAO, Symposium international Horticulture urbaine et périurbaine au "siécle des villes". Enseignements, enjeux et opportunités, Dakar, Sénégal. <hal-01757784>. (2010).

[13] O. Touré et S. M. Seck. Exploitations familiales et entreprises agricoles dans la zone des Niayes au Sénégal. International Institute for Environment and Development. Vol. 133, 66 P. 2005.

[14] K. Diop, C. A. T. Faye et S. A. Sow. « La Grande Niaye de Pikine, un espace humide à haute valeur agronomique au cœur de l'agglomération urbaine de Dakar : analyse des enjeux socioéconomiques ». 2019. 